\title{
Epidemiological Investigation, Prevalence \& Antibiogram Study of Potential Zoonotic Bacterial Pathogens of Household Pets at Dinajpur District of Bangladesh
}

\author{
Samina Akter \\ Department of Microbiology \\ Hajee Mohammad Danesh Science and Technology \\ University, Dinajpur, Bangladesh.
} conducted to ascertain the prevalence of potential zoonotic bacterial pathogen in household pet animals (dog, cat and rabbit). The study was done in selected areas of Dinajpur district during the period of July 2016 to June 2017. A total of 79 pet animals comprising of $\mathbf{5 0}$ $(63.29 \%)$ dogs; $10(12.66 \%)$ cats and $19(24.05 \%)$ rabbits were observed and samples were collected considering different socio demographic variable. The organisms were isolated by using standard microbiological techniques. A total of 404 samples were examined and 7 isolates of potential zoonotic bacterial pathogens were isolated in pet animals. The overall prevalence of zoonotic pathogens in dogs out of 250 samples, was $15.2 \% \quad E$. coli; $10.8 \%$ Klebsiella spp; $12 \%$ Salmonella spp; 8\% Proteus spp; 8.8\% Pseudomonas spp; 12\% Staphylococcus spp; 10.4\% Streptococcus spp respectively. The overall prevalence of zoonotic pathogens in cats out of 40 samples was $17.5 \%$ E.coli; $10 \%$ Klebsiella spp; $12.5 \%$ Salmonella spp; $10 \%$ Proteus spp; 10\% Pseudomonas spp; 15\% Staphylococcus spp; $15 \%$ Streptococcus spp respectively. The overall prevalence of zoonotic pathogens in rabbits was $12.28 \%$ E.coli; $\mathbf{7 . 8 9 \%}$ Klebsiella spp; $9.65 \%$ Salmonella spp; $4.38 \% \quad$ Proteus spp; $1.75 \%$ Pseudomonas spp; 6.14\% Staphylococcus spp; 6.14\% Streptococcus spp respectively. The prevalence of bacterial zoonotic pathogen between pet animals and housing system, hygienic condition, vaccination were statistically significant $(P \leq 0.01)$. On the other hand the prevalence of bacterial zoonotic pathogen between pet animals and age, sex, breed, body weight, diet, educational status of pet owners were not statistically significant ( $P>\quad 0.05)$. On antibiogram study 18 antibiotics were used for antimicrobial sensitivity test. Gram negative isolates were more sensitive against Chloramphenicol, Cephalexin, Gentamycin and Kanamycin. On the other hand gram positive isolates were more sensitive against Azithromycin, Levofloxacin, Colistin and Gentamycin. The isolates were highly resistant against Amoxycillin, Bacitracin, Penicillin and Vancomycin.

Keywords:- Zoonotic Discease, Zoonotic Bacterial Pathogens, Statistically Study, Antibiogram Study.

\author{
Dr. Md. Atiqul Haque; Israt Jahan \\ Department of Microbiology \\ Hajee Mohammad Danesh Science and Technology \\ University, Dinajpur, Bangladesh.
}

\section{INTRODUCTION}

Animal domestication has spread from long ago, the number of households keeping animals has increased and the relationship between human and animals has become closer under the life environment in contemporary society where technology has developed [1]. Our cave dwelling ancestors used dogs as the co-partner in hunting job. Subsequently, in all civilization dogs were used as guards, companions and hunters and in times of war. So, first pet animal of man was dogs and the relationship between human and dogs began 12,000-15,000 years ago and with cats nearly 5,000 years ago [2].

Dogs and cats have significant benefits to our society like companionship, play with children, guard the house and from any adverse condition alert the owner, used as gift to special one and economic purpose [3]. In many households contributing to the physical, social and mental development of children and the well-being of their owners, they act as important companion [4] [5].

Now-a-days, rabbits are the popular pets, coming third after dogs and cats and also still play an important role in the industrial sector and as laboratory animals [6]. Rabbits make excellent pets because they are clean, docile and calm by nature [7]. They are extremely delicate animals and are prone to many bacterial and fungal diseases if proper care is not taken and can also result in rabbit malnutrition, growth retardation, feed remuneration reduction and even death [8].

Pet animals kept for pleasures and companionship are usually domesticated and selectively bred for coexistence with human beings, besides their value as pets, pets serve utilitarian purposes protecting homes and property, destroying vermin and providing means of transport. They have been sharing our environment and have gained a major status as "pets" in our modern, very urbanized society. In the middle of the 20th century, they are more and more considered as "family members" within households; not to mention sometimes as substitutes for children [9]. 
Diseases and infections those are naturally transmitted between vertebrate animals and man are called zoonoses [10]. There are approximately 1415 pathogens known to affect humans of which about $61 \%$ of all human pathogens are zoonotic [11]. Household pets (dog, cat \& rabbit), defined here as any animals kept within households by people for company, enjoyment, work or psychological support, can be colonized or infected with a wide variety of bacteria and fungi pathogenic to animals and people. Petassociated bacterial and fungal zoonoses represent a relatively neglected area compared with food borne zoonoses [12].

In our country dogs, cats and rabbits are often purchased as a pet and these house-hold pets appear to be an important source of zoonotic diseases. Consequent to their popularity pet owners become increasingly knowledgeable and are willing to pay for advanced treatment and diagnostics [13]. Together with the increasing concern in the private, scientific and industrial sector to keep the animal healthy also the need regarding veterinarian expertise increased. Therefore, control of zoonotic diseases is even more important due to the increasing number of immunocompromised people but the distribution of pets around the world and their differences modify their role in zoonotic disease transmission. The risk of pathogen transmission from pet to the owner is relatively small, when simple precautions are taken. Therefore, the role of veterinarians is essential since they have to provide pet owners with accurate information [14]. Continuous investigative research provides new insights in clinical patterns, symptoms, etiologies and pathogenesis of different zoonotic infections/ diseases having public health significance to improve our knowledge of understanding.

\section{METARIALS AND METHODS}

The present study was carried out of pet animals (dog, cat and rabbit) under the sadar of Dinajpur district and samples (oral swab, skin scrapping, nasal swab, rectal swab, feaces and urine) were taken in the bacteriology laboratory of the department of Microbiology, Hajee Mohammad Danesh Science and Technology University, Dinajpur, Bangladesh for the identification of bacteria \& fungus by different microbiological methods.

The study was directed during the period from July 2016 to June 2017.

\section{A. Plan of the experiment work at a glance}

All of those samples were collected from different areas of Dinajpur sadar with a thermo flask containing ice in suitable diluent viz peptone water maintaining aseptic condition. Then all of the samples were transferred to the microbiological laboratory of department of Microbiology, HSTU, Dinajpur, Bangladesh. Appropriate amount of samples were primarily inoculated into Nutrient agar and Plate count agar for determining the density of bacterial profiles in pet animals that they carry and obtained their total viable count (TVC). Subsequently Nutrient agar, Blood agar, EMB agar, SS agar, MacConkey agar,
Cetrimide agar and Staphylococcus agar NO. 110 base were employed and specific biochemical tests were done for isolation and identification of bacteria. At last performed antibiotic sensitivity test with the pure isolated bacteria.

\section{B. Methods}

\section{$>$ Questionnaire}

A structured pre-coded questionnaire focusing on the information of pet animals and pet owners related to the transmission of zoonotic infection was written in English and converted to Bengali whenever needed during data collection. Socio-demographic variables of the study population such as age, sex, breed, body weight, housing system, hygienic condition, vaccination and education level of pets owner. Owner of pets in the study were interviewed by direct contact, visited their home or a few times by phone.

\section{$>$ Collection and transportation of Samples}

A total of 404 samples (oral swab, nasal swab, skin scrapping, rectal swab, feaces, and urine) were collected from pet animals (dog, cat, rabbit) by sterile cotton buds and took into sterile tube containing with $1 \%$ peptone water. Each sample was marked properly with date, time and sample number then kept in an insulated ice box. After collection of those samples in a tube closed the cap and taken to the laboratory for microbiological investigation.

All samples were brought to the laboratory within half an hour of collection and subjected to bacteriological examination. Samples were kept under refrigeration at $4^{0}$ $7^{0} \mathrm{C}$ until study.

\section{> Sampling and Processing of Samples}

Proper care was taken during the sampling procedure to prevent contamination of sample. The samples tubes were completely tied at the time of sampling that prevent contamination. After came to the laboratory the sample tubes were shake to mix with $1 \%$ peptone water. After that 9ml PBS (Phosphate Buffer Solution) was taken each test tube for serial dilution. Then $1 \mathrm{ml}$ sample was taken from each sample for ten-fold serial dilution $\left(10^{-1}\right.$ to $\left.10^{-10}\right)$ and $50 \mu \mathrm{l}$ samples were seeded on nutrient agar and plate count agar using spread plate method. The plating was done in the laminar flow to maintain aseptic conditions and the medium were then incubated at $37^{\circ} \mathrm{C}$ for $24 \mathrm{~h}$.

\section{> Microbial assessment of the collected samples}

Samples were collected and each of the samples was diluted with distilled water as $10^{-1}$ to $10^{-10}$. Then $50 \mu \mathrm{l}$ samples were taken and spread in plate count agar(PCA) plate following the spread-plate method and incubated at $37^{\circ} \mathrm{C}$ for $24 \mathrm{~h}$. The number of organisms per $\mathrm{ml}$ or per gram of original culture was calculated by multiplying the number of colonies counted by the dilution factor: Number of cells per $\mathrm{ml}$ or per gram $=$ number of colonies $\times$ Dilution factor/Volume of dilution. 


\section{Identification of the bacterial genera}

The bacterial isolates were transferred to sterilized plates for purification and identification. The grown bacteria were smear on a slide, stained with gram's stain solution to detect bacterial structures, examined under microscope (100X) and identified on the basis of their colony morphology then confirmed by biochemical test.

\section{Antibiotic Sensitivity Test}

The antibiotic resistance was determined by KirbyBauer disc diffusion technique using Mueller-Hinton agar (Difco), according to the recommendations of National Committee for Clinical Laboratory Standards [15]. After overnight incubation at $37{ }^{\circ} \mathrm{C}$, the diameter in millimeters of the zones of inhibition around each of the antimicrobial discs was recorded and categorized as resistant or sensitive in accordance with company recommendations. All isolates were tested for sensitivities to 18 of routine and practical antibiotics.

\section{Statistical Analysis}

Data were analyzed using SPSS for Windows (version 21.0). Prevalence of bacterial isolates was expressed in simple descriptive statistics such as means and standard deviation. For CFU/gm values, one-way ANOVA test and the detection of significant differences between $(p \leq 0.05)$ socio demographic variable and zoonotic pathogens Chisquare test $\left(\chi^{2}\right)$ was done.

\section{RESULTS}

Socio demographic variable of study population with (dog, cat and rabbit) are as follows in TABLE 1 (A); 1 (B); 1 (C).

\begin{tabular}{|c|c|c|c|}
\hline \multicolumn{2}{|c|}{ Socio demographic Profile } & \multirow{2}{*}{$\begin{array}{c}\text { Frequency } \\
5\end{array}$} & \multirow{2}{*}{$\begin{array}{c}\text { Percentage (\%) } \\
10\end{array}$} \\
\hline Age & Young (<6 months) & & \\
\hline & Adult (7-20 months) & 15 & 30 \\
\hline & Old (>21months) & 30 & 60 \\
\hline \multirow[t]{2}{*}{ Sex } & Male & 24 & 48 \\
\hline & Female & 26 & 52 \\
\hline \multirow[t]{2}{*}{ Breed } & Indigenous & 23 & 46 \\
\hline & Exotic & 27 & 54 \\
\hline \multirow[t]{3}{*}{ Body wt. } & $<7 \mathrm{~kg}$ & 5 & 10 \\
\hline & $8-17 \mathrm{~kg}$ & 17 & 34 \\
\hline & $>18 \mathrm{~kg}$ & 28 & 56 \\
\hline \multirow[t]{3}{*}{ Housing System } & Poor & 20 & 40 \\
\hline & Good & 21 & 42 \\
\hline & Excellent & 9 & 18 \\
\hline \multirow[t]{3}{*}{ Diet } & Ready Feed & 0 & 0 \\
\hline & Raw Food & 38 & 76 \\
\hline & Both & 12 & 24 \\
\hline \multirow[t]{3}{*}{ Hygienic Condition } & Poor & 15 & 30 \\
\hline & Good & 28 & 56 \\
\hline & Excellent & 7 & 14 \\
\hline \multirow[t]{2}{*}{ Vaccination } & Yes & 27 & 54 \\
\hline & No & 23 & 46 \\
\hline \multirow[t]{3}{*}{ Education level of pets owner } & Under Graduate & 20 & 40 \\
\hline & Graduate & 12 & 24 \\
\hline & Post Graduate & 18 & 36 \\
\hline \multicolumn{2}{|c|}{ Total } & 50 & 100 \\
\hline
\end{tabular}

Table 1 (A):- Socio demographic variable of study population (in case of dog) 


\begin{tabular}{|c|c|c|c|}
\hline \multicolumn{2}{|c|}{ Socio demographic Profile } & Frequency & Percentage (\%) \\
\hline \multirow[t]{3}{*}{ Age } & Young (<6 months) & 2 & 20 \\
\hline & Adult (7-12 months) & 4 & 40 \\
\hline & Old (>13months) & 4 & 40 \\
\hline \multirow[t]{2}{*}{ Sex } & Male & 2 & 20 \\
\hline & Female & 8 & 80 \\
\hline \multirow[t]{2}{*}{ Breed } & Indigenous & 10 & 100 \\
\hline & Exotic & 0 & 0 \\
\hline \multirow[t]{3}{*}{ Body wt. } & $<1 \mathrm{~kg}$ & 2 & 20 \\
\hline & $1-3 \mathrm{~kg}$ & 4 & 40 \\
\hline & $>3 \mathrm{~kg}$ & 4 & 40 \\
\hline \multirow[t]{3}{*}{ Housing System } & Poor & 8 & 80 \\
\hline & Good & 2 & 20 \\
\hline & Excellent & 0 & 0 \\
\hline \multirow[t]{3}{*}{ Diet } & Ready Feed & 0 & 0 \\
\hline & Raw Food & 10 & 100 \\
\hline & Both & 0 & 0 \\
\hline \multirow[t]{3}{*}{ Hygienic Condition } & Poor & 8 & 80 \\
\hline & Good & 2 & 20 \\
\hline & Excellent & 0 & 0 \\
\hline \multirow[t]{2}{*}{ Vaccination } & Yes & 0 & 0 \\
\hline & No & 10 & 100 \\
\hline \multirow[t]{3}{*}{ Education level of pets owner } & Under Graduate & 2 & 20 \\
\hline & Graduate & 8 & 80 \\
\hline & Post Graduate & 0 & 0 \\
\hline \multicolumn{2}{|c|}{ Total } & 10 & 100 \\
\hline
\end{tabular}

Table 1 (B): Socio demographic variable of study population (in case of cat)

\begin{tabular}{|c|c|c|c|}
\hline \multicolumn{2}{|c|}{ Socio demographic Profile } & Frequency & Percentage (\%) \\
\hline \multirow[t]{3}{*}{ Age } & Young (<6 months) & 2 & 10.53 \\
\hline & Adult (7-30 months) & 10 & 52.63 \\
\hline & Old (>31months) & 7 & 36.84 \\
\hline \multirow[t]{2}{*}{ Sex } & Male & 11 & 57.89 \\
\hline & Female & 8 & 42.11 \\
\hline \multirow[t]{2}{*}{ Breed } & Indigenous & 19 & 100 \\
\hline & Exotic & 0 & 0 \\
\hline \multirow[t]{3}{*}{ Body wt. } & $<1 \mathrm{~kg}$ & 2 & 10.53 \\
\hline & $1-2 \mathrm{~kg}$ & 11 & 57.89 \\
\hline & $>2 \mathrm{~kg}$ & 6 & 31.58 \\
\hline \multirow[t]{3}{*}{ Housing System } & Poor & 4 & 21.05 \\
\hline & Good & 15 & 78.95 \\
\hline & Excellent & 0 & 0 \\
\hline \multirow[t]{3}{*}{ Diet } & Ready Feed & 0 & 0 \\
\hline & Raw Food & 7 & 36.84 \\
\hline & Both & 12 & 63.16 \\
\hline \multirow[t]{3}{*}{ Hygienic Condition } & Poor & 4 & 21.05 \\
\hline & Good & 15 & 78.95 \\
\hline & Excellent & 0 & 0 \\
\hline \multirow{3}{*}{$\begin{array}{c}\text { Education level of pets } \\
\text { owner }\end{array}$} & Under Graduate & 5 & 26.31 \\
\hline & Graduate & 2 & 10.53 \\
\hline & Post Graduate & 12 & 63.16 \\
\hline \multicolumn{2}{|c|}{ Total } & 19 & 100 \\
\hline
\end{tabular}

Table 1 (C): Socio demographic variable of study population (in case of rabbit) 


\section{A. Prevalence and Identification of Zoonotic Bacterial Pathogen}

Results of Total Bacterial Count (TBC)

TBC expressed as CFU/g (Colony Forming Unit per gram) of different samples from household pet animals were shown in TABLE 2. In case of dogs the average colony counts were $10.14 \pm .76$ for oral swab, $10.16 \pm .78$ for skin scrapping, $10.14 \pm .75$ for

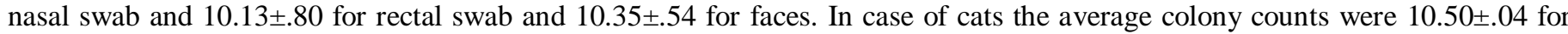
oral swab, $10.57 \pm .057$ for skin scrapping, $10.55 \pm .11$ for nasal swab and $10.63 \pm .15$ for rectal swab. In case of rabbits the average

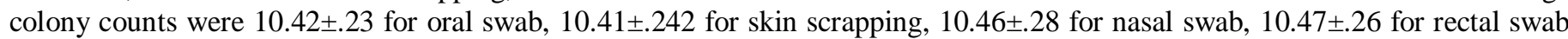
and $10.45 \pm .29$ for faces and $10.44 \pm .25$ for urine.

\begin{tabular}{|c|c|c|c|c|}
\hline \multirow{8}{*}{$\begin{array}{c}\text { TBC }(\text { mean } \pm \text { SD }) \\
\text { CFU/g }\end{array}$} & & \multicolumn{3}{|c|}{ Species } \\
\hline & & Dog & Cat & Rabbit \\
\hline & Oral swab & $10.14 \pm .76$ & $10.50 \pm .04$ & $10.42 \pm .23$ \\
\hline & Skin scrapping & $10.16 \pm .78$ & $10.57 \pm .06$ & $10.41 \pm .242$ \\
\hline & Nasal secretion & $10.14 \pm .75$ & $10.56 \pm .11$ & $10.46 \pm .28$ \\
\hline & Rectal swab & $10.13 \pm .80$ & $10.63 \pm .26$ & $10.47 \pm .26$ \\
\hline & Feaces & $10.35 \pm .54$ & ND & $10.45 \pm .29$ \\
\hline & Urine & ND & ND & $10.44 \pm .25$ \\
\hline \multicolumn{2}{|c|}{ P- Value } & 0.51 & 0.10 & 0.081 \\
\hline
\end{tabular}

Table 2:- TBC of potentially zoonotic bacterial pathogen in dog, cat, and rabbit.

[All values are converted into logarithms 10; All counts are expressed in colony forming units (cfu); ND= Not Done; SD= Standard Division.]

Figure 1. Summarized the prevalence of zoonotic bacterial pathogen in pet dogs, cat \& rabbit. In dog the overall prevalence of E.coli; Klebsiella spp; Salmonella spp; Proteus spp; Pseudomonas spp; Staphylococcus spp and Streptococcus spp were $15.2 \% ; 10.8 \% ; 12 \% ; 8 \% ; 8.8 \% ; 12.8 \%$ and $10.4 \%$ respectively. In cat the overall prevalence of E.coli; Klebsiella spp; Salmonella spp; Proteus spp; Pseudomonas spp; Staphylococcus spp and Streptococcus spp were 17.5\%; 10\%; 12.5\%; 10\%; 10\%; 15\% and $15 \%$ respectively. And in rabbit the overall prevalence of E.coli; Klebsiella spp; Salmonella spp; Proteus spp; Pseudomonas spp; Staphylococcus spp and Streptococcus spp were 12.28\%; 7.89\%; 11\%; 5\%; 2\%; 6.14\% and 6.14\% respectively.

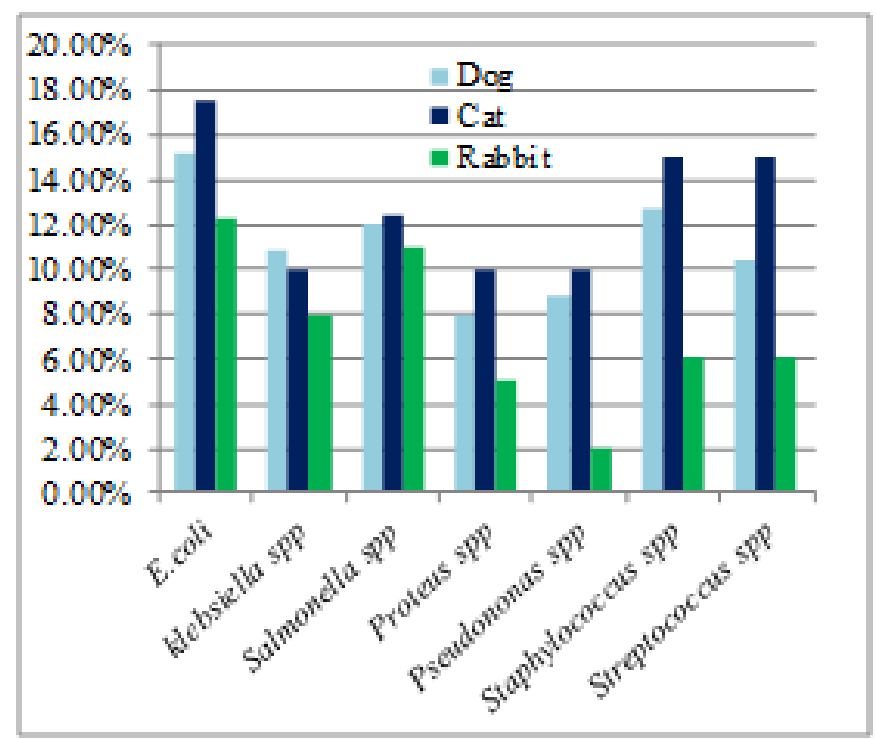

Fig 1:- Prevalence of potentially bacterial zoonotic pathogens in Dog, Cat \& Rabbit. 


\section{B. Results of Cultural Examination}

The cultural characteristics of E. coli, Klebsiella spp, Salmonella spp, Proteus spp, Pseudomonas spp, Staphylococcus spp, and Streptococcus spp on various media are presented in Table 3.

\begin{tabular}{|c|c|c|c|c|}
\hline $\mathbf{S} / \mathbf{N}$ & Name of bacteria & $\begin{array}{l}\text { Staining charact- } \\
\text { eristic }\end{array}$ & Name of media & Colony characteristics \\
\hline \multirow[t]{4}{*}{01} & \multirow[t]{4}{*}{ E. coli } & \multirow{4}{*}{$\begin{array}{l}\text { Gram negative large } \\
\text { rod shaped pink } \\
\text { colour. }\end{array}$} & NA & Large, mucoid, white colony. \\
\hline & & & $\mathrm{MaC}$ & $\begin{array}{l}\text { Produce large mucoid rose pink } \\
\text { colony }\end{array}$ \\
\hline & & & EMB agar & $\begin{array}{l}\text { Transmitted light blue-black } \\
\text { center with a narrow, clear edge. } \\
\text { Blue-green metallic sheen with } \\
\text { reflected light. }\end{array}$ \\
\hline & & & BGA & Yellow-green colony. \\
\hline \multirow[t]{3}{*}{02} & \multirow[t]{3}{*}{ Klebsiella spp } & \multirow{3}{*}{$\begin{array}{l}\text { Gram negative rod } \\
\text { shaped pink colour. }\end{array}$} & NA & Large colony. \\
\hline & & & $\mathrm{MaC}$ & Large, red, mucoid \\
\hline & & & EMB agar & $\begin{array}{l}\text { Mucoid, no metallic sheen. With } \\
\text { transmitted light, gray-brown } \\
\text { centers and pink color with clear } \\
\text { edges. }\end{array}$ \\
\hline \multirow[t]{4}{*}{03} & \multirow[t]{4}{*}{ Salmonella spp. } & \multirow{4}{*}{$\begin{array}{l}\text { Gram negative small } \\
\text { rod shaped pink } \\
\text { colour. }\end{array}$} & NA & $\begin{array}{l}\text { Smooth. Opaque, translucent } \\
\text { colonies. }\end{array}$ \\
\hline & & & SS Agar & $\begin{array}{l}\text { Opaque, smooth, round with black } \\
\text { centered colonies. }\end{array}$ \\
\hline & & & $\mathrm{MaC}$ & $\begin{array}{l}\text { Small, white, translucent dew } \\
\text { drop like colonies. }\end{array}$ \\
\hline & & & BGA & $\begin{array}{c}\text { Good growth red and pink white } \\
\text { colonies. }\end{array}$ \\
\hline \multirow[t]{3}{*}{04} & \multirow[t]{3}{*}{ Proteus spp } & \multirow[t]{3}{*}{$\begin{array}{l}\text { Gram negative small } \\
\text { rod shaped. }\end{array}$} & NA & $\begin{array}{l}\text { Circular, smooth, entire, opaque } \\
\text { with white color colonies. }\end{array}$ \\
\hline & & & $\mathrm{MaC}$ & $\begin{array}{c}\text { Colourless and transparent } \\
\text { colonies. } \\
\end{array}$ \\
\hline & & & BGA & $\begin{array}{l}\text { Colonies of a pale pink color, } \\
\text { transparent and surrounded by a } \\
\text { brilliant red halo. }\end{array}$ \\
\hline \multirow[t]{4}{*}{05} & \multirow[t]{4}{*}{ Pseudomonas spp } & \multirow{4}{*}{$\begin{array}{l}\text { Gram negative small } \\
\text { rod shaped pink } \\
\text { colour. }\end{array}$} & NA & $\begin{array}{l}\text { Large, smooth, low convex and } \\
\text { greenish pigment with fruity odor. }\end{array}$ \\
\hline & & & $\mathrm{MaC}$ & $\begin{array}{l}\text { Pale colour flat non lactose } \\
\text { fermenting colonies }\end{array}$ \\
\hline & & & $\mathrm{CA}$ & Colonies are greenish in color. \\
\hline & & & $\mathrm{BA}$ & $\beta$-hemolytic colonies. \\
\hline \multirow[t]{5}{*}{06} & \multirow[t]{5}{*}{ Staphylococcus spp. } & \multirow{5}{*}{$\begin{array}{l}\text { Gram positive } \\
\text { cluster liked violet } \\
\text { colour. }\end{array}$} & NA & $\begin{array}{l}\text { Black colour/ non-colour smooth, } \\
\text { glistening colonies. }\end{array}$ \\
\hline & & & NB & Uniform turbidity. \\
\hline & & & MSA & Yellow colonies. \\
\hline & & & SA No.110 & Yellow colonies. \\
\hline & & & $\mathrm{BA}$ & $\beta$-hemolytic colonies. \\
\hline \multirow[t]{4}{*}{07} & \multirow[t]{4}{*}{ Streptococcus spp } & \multirow{4}{*}{$\begin{array}{l}\text { Gram positive short } \\
\text { chain shaped violet } \\
\text { colour. }\end{array}$} & NA & Uniform turbidity. \\
\hline & & & NB & Moderate growth. \\
\hline & & & MSA & Pink colony \\
\hline & & & $\mathrm{BA}$ & $\begin{array}{l}\text { Small, dry colony surrounded by } \\
\beta \text {-hemolysis. }\end{array}$ \\
\hline
\end{tabular}

Table 3:- The result of cultural characteristics of the bacteria which are isolated from different samples of pet animals (dog, cat and rabbit).

[Where; NA = Nutrient Agar; NB = Nutrient Broth; MaC = Mac-Conkey's Agar; EMB = Eosin Methylene Blue; BGA = Brilliant Green Agar; SS = Salmonella-Shegilla Agar; CA = Cetrimide agar; BA = Blood Agar; MSA = Mannitol Salt Agar; SA No. $110=$ Staphylococcus Agar No. 110] 


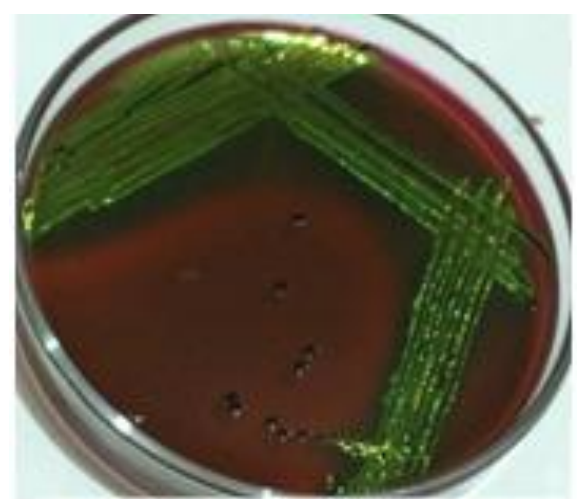

Fig 2:- E.coli on Eosin Methylene Blue agar

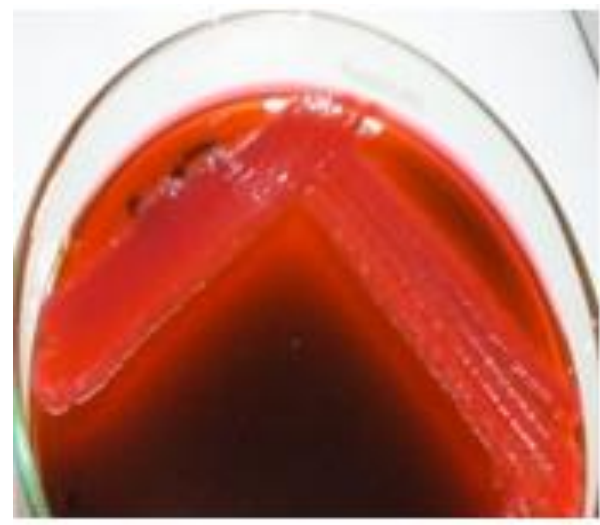

Fig 3:- klebsiella spp on Eosin Methylene Blue agar

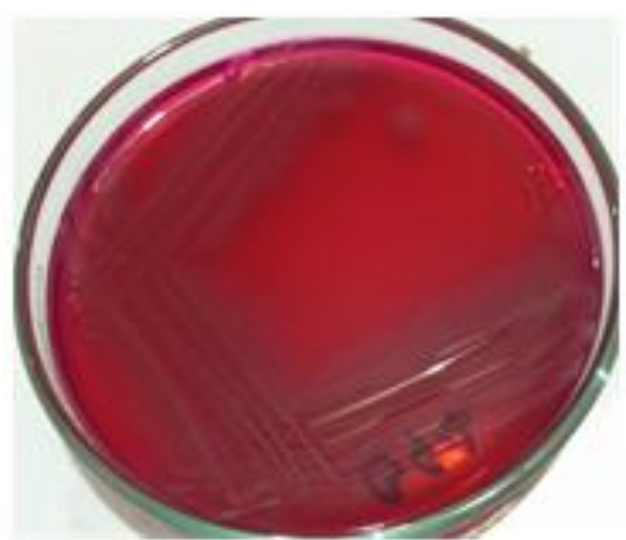

Fig 4:- Salmonella spp on Brilliant Green Agar

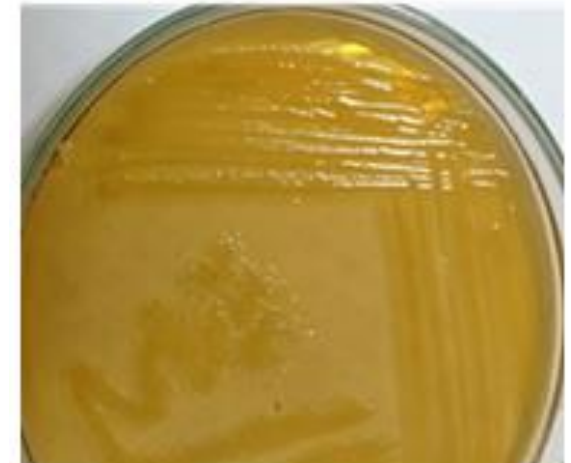

Fig 5:- Proteus spp on Mac-Conkey Agar

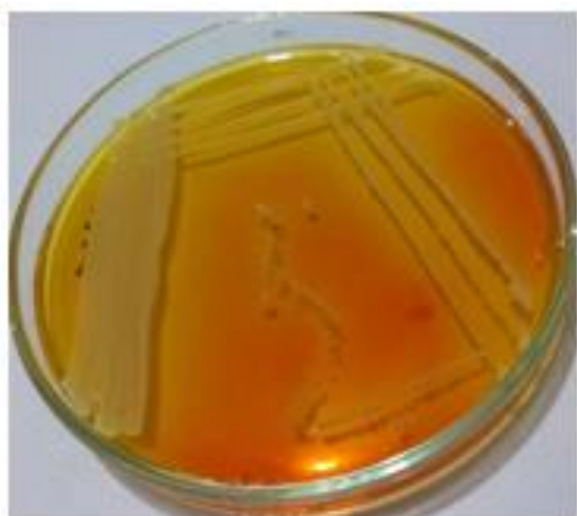

Fig 6:- Staphylococcus spp on Mannitol Salt

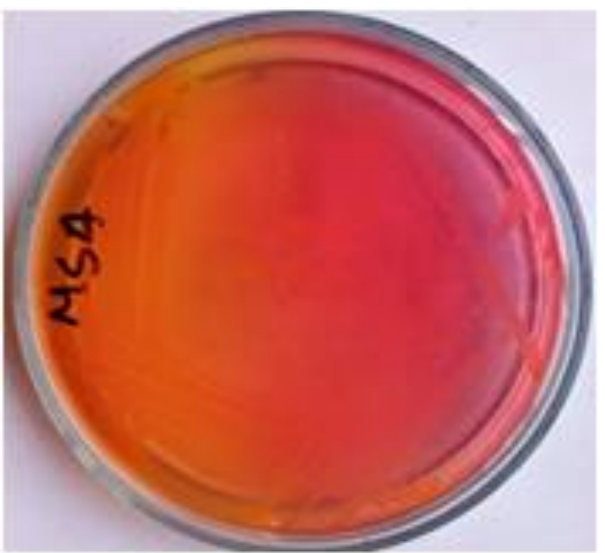

Fig 7:- Streptococcus spp on Mannitol Salt Agar 
ISSN No:-2456-2165

\begin{tabular}{|c|c|c|c|c|c|c|c|c|}
\hline Species & \multirow[t]{2}{*}{ Sex } & \multirow[t]{2}{*}{ Age } & \multicolumn{6}{|c|}{ TBC $($ mean \pm SD) CFU $/ g$} \\
\hline \multirow{9}{*}{$\operatorname{Dog}(n=50)$} & & & OS & SS & NS & $\mathbf{R S}$ & $\mathbf{F}$ & Urine \\
\hline & $\begin{array}{c}\text { Male } \\
(\mathrm{N}=24)\end{array}$ & $\begin{array}{l}\text { Young } \\
(<6 \mathrm{M})\end{array}$ & $\begin{array}{c}9.05 \\
\pm 2.22\end{array}$ & $\begin{array}{c}9.66 \\
\pm 1.15\end{array}$ & $\begin{array}{c}9.16 \\
\pm 2.06\end{array}$ & $\begin{array}{c}8.69 \\
\pm 2.81\end{array}$ & $\begin{array}{c}9.21 \\
\pm 2.07\end{array}$ & ND \\
\hline & & $\begin{array}{c}\text { Adult } \\
(7-20 \mathrm{M})\end{array}$ & $\begin{array}{l}10.33 \\
\pm 0.36\end{array}$ & $\begin{array}{l}10.13 \\
\pm 0.79\end{array}$ & $\begin{array}{l}10.22 \\
\pm 0.36\end{array}$ & $10.27 \pm 0.35$ & $\begin{array}{l}10.33 \\
\pm 0.32\end{array}$ & ND \\
\hline & & $\begin{array}{c}\text { Old } \\
(>21 \mathrm{M})\end{array}$ & $\begin{array}{c}9.99 \\
\pm 0.94\end{array}$ & $\begin{array}{l}10.03 \\
\pm 1.17\end{array}$ & $\begin{array}{c}9.93 \\
\pm 0.62\end{array}$ & $9.88 \pm 0.95$ & $\begin{array}{l}10.29 \\
\pm 0.99\end{array}$ & ND \\
\hline & & P- Value & 0.187 & 0.818 & 0.316 & 0.148 & 0.065 & ND \\
\hline & Female(N=26) & $\begin{array}{l}\text { Young } \\
(<6 \mathrm{M})\end{array}$ & $\begin{array}{c}9.76 \\
\pm 0.49\end{array}$ & $\begin{array}{c}9.83 \\
\pm 0.48\end{array}$ & $\begin{array}{l}10.07 \\
\pm 0.46\end{array}$ & $9.998 \pm 0.43$ & $\begin{array}{l}10.31 \\
\pm 0.25\end{array}$ & ND \\
\hline & & $\begin{array}{c}\text { Adult } \\
(7-20 \mathrm{M})\end{array}$ & $\begin{array}{l}10.52 \\
\pm 0.25\end{array}$ & $\begin{array}{l}10.56 \\
\pm 0.22\end{array}$ & $\begin{array}{l}10.51 \\
\pm 0.18\end{array}$ & $10.47 \pm 0.28$ & $\begin{array}{l}10.61 \\
\pm 0.12\end{array}$ & ND \\
\hline & & $\begin{array}{c}\text { Old } \\
(>21 \mathrm{M})\end{array}$ & $\begin{array}{l}10.13 \\
\pm 0.74\end{array}$ & $\begin{array}{l}10.18 \\
\pm 0.67\end{array}$ & $\begin{array}{l}10.17 \\
\pm 0.75\end{array}$ & $10.21 \pm 0.63$ & $\begin{array}{l}10.41 \\
\pm 0.46\end{array}$ & ND \\
\hline & & P- Value & 0.25 & 0.201 & 0.495 & 0.465 & 0.469 & ND \\
\hline \multirow{8}{*}{$\begin{array}{c}\text { Cat } \\
(\mathbf{n}=\mathbf{1 0})\end{array}$} & \multirow[t]{4}{*}{$\begin{array}{l}\text { Male } \\
(\mathrm{N}=2)\end{array}$} & $\begin{array}{l}\text { Young } \\
(<6 \mathrm{M})\end{array}$ & NB & NB & NB & NB & ND & ND \\
\hline & & $\begin{array}{c}\text { Adult } \\
(7-12 \mathrm{M})\end{array}$ & $\begin{array}{l}10.53 \\
\pm 0.08 \\
\end{array}$ & $\begin{array}{l}10.53 \\
\pm 0.08 \\
\end{array}$ & $\begin{array}{l}10.75 \\
\pm 0.02 \\
\end{array}$ & $10.74 \pm 0.01$ & $\mathrm{ND}$ & ND \\
\hline & & $\begin{array}{c}\text { Old } \\
(>13 \mathrm{M})\end{array}$ & NB & NB & $\mathrm{NB}$ & NB & $\mathrm{ND}$ & ND \\
\hline & & P-Value & 0.003 & 0.003 & 0.001 & 0.00 & ND & ND \\
\hline & \multirow[t]{4}{*}{ Female(N=8) } & $\begin{array}{l}\text { Young } \\
(<6 \mathrm{M})\end{array}$ & NB & NB & NB & NB & ND & ND \\
\hline & & $\begin{array}{c}\text { Adult } \\
(7-12 \mathrm{M}) \\
\end{array}$ & $\begin{array}{l}10.51 \\
\pm 0.05 \\
\end{array}$ & $\begin{array}{l}10.52 \\
\pm 0.05 \\
\end{array}$ & $\begin{array}{l}10.48 \\
\pm 0.02 \\
\end{array}$ & $10.45 \pm 0.02$ & ND & ND \\
\hline & & $\begin{array}{c}\text { Old } \\
(>13 \mathrm{M})\end{array}$ & NB & NB & NB & NB & ND & ND \\
\hline & & P- Value & 0.000 & 0.000 & 0.000 & 0.000 & ND & ND \\
\hline \multirow{8}{*}{ Rabbit(n=19) } & \multirow[t]{4}{*}{$\begin{array}{c}\text { Male } \\
(\mathrm{N}=11)\end{array}$} & $\begin{array}{l}\text { Young } \\
(<6 \mathrm{M})\end{array}$ & $\begin{array}{l}10.59 \\
\pm 0.17\end{array}$ & $\begin{array}{l}10.64 \\
\pm 0.22\end{array}$ & $\begin{array}{c}10.69 \\
\pm 0.1\end{array}$ & $10.52 \pm 0.39$ & $\begin{array}{l}10.72 \\
\pm 0.06\end{array}$ & $10.26 \pm 0.18$ \\
\hline & & $\begin{array}{c}\text { Adult } \\
(7-30 \mathrm{M})\end{array}$ & $\begin{array}{l}10.42 \\
\pm 0.17 \\
\end{array}$ & $\begin{array}{l}10.43 \\
\pm 0.18 \\
\end{array}$ & $\begin{array}{l}10.42 \\
\pm 0.31 \\
\end{array}$ & $10.44 \pm 0.26$ & $\begin{array}{l}10.42 \\
\pm 0.29 \\
\end{array}$ & $10.51 \pm 0.12$ \\
\hline & & $\begin{array}{c}\text { Old } \\
(>31 \mathrm{M})\end{array}$ & $\begin{array}{l}10.39 \\
\pm 0.16 \\
\end{array}$ & $\begin{array}{l}10.49 \\
\pm 0.02 \\
\end{array}$ & $\begin{array}{l}10.53 \\
\pm 0.18 \\
\end{array}$ & $10.52 \pm 0.25$ & $\begin{array}{l}10.22 \\
\pm 0.22 \\
\end{array}$ & $10.51 \pm 0.17$ \\
\hline & & P- Value & 0.36 & 0.33 & 0.42 & 0.85 & 0.17 & 0.098 \\
\hline & \multirow[t]{4}{*}{ Female $(\mathrm{N}=8)$} & $\begin{array}{l}\text { Young } \\
(<6 \mathrm{M})\end{array}$ & NB & $\mathrm{NB}$ & NB & NB & NB & NB \\
\hline & & $\begin{array}{c}\text { Adult } \\
(7-30 \mathrm{M})\end{array}$ & $\begin{array}{l}10.53 \\
\pm 0.37 \\
\end{array}$ & $\begin{array}{l}10.48 \\
\pm 0.39 \\
\end{array}$ & $\begin{array}{l}10.55 \\
\pm 0.37 \\
\end{array}$ & $10.59 \pm 0.34$ & $\begin{array}{l}10.61 \\
\pm 0.35 \\
\end{array}$ & $10.52 \pm 0.39$ \\
\hline & & $\begin{array}{c}\text { Old } \\
(>31 \mathrm{M})\end{array}$ & NB & NB & NB & NB & NB & NB \\
\hline & & P- Value & 0.000 & 0.000 & 0.000 & 0.000 & 0.000 & 0.000 \\
\hline
\end{tabular}

Table 4:- Species; sex \& age wise distribution of total viable count (TVC) of zoonotic bacterial pathogen from dog; cat; rabbit.

[WHERE; All values are converted into logarithms 10; All counts are expressed in colony forming units (CFU); $\mathrm{M}=$ Months; ND= Not Done; NB= Nobody; SD = Standard Division; SS=Skin Scrapping; NS = Nasal Secretion; RS=Rectal Swab; OS = Oral Swab; F = Feaces $]$ 
ISSN No:-2456-2165

\begin{tabular}{|c|c|c|c|}
\hline Breed & Samples & TBC (mean+ SD) CFU/g & P- Value \\
\hline \multirow[t]{5}{*}{ Local/ Indigenous $(\mathrm{n}=23)$} & Oral swab & $9.99 \pm 0.77$ & \multirow{5}{*}{.0001} \\
\hline & Skin scrapping & $9.99 \pm 0.86$ & \\
\hline & Nasal secretion & $10.14 \pm 0.83$ & \\
\hline & Rectal swab & $2.33 \pm 1.57$ & \\
\hline & Feaces & $10.30 \pm 0.65$ & \\
\hline \multirow[t]{5}{*}{ Exotic $(\mathrm{n}=27)$} & Oral swab & $10.25 \pm 0.75$ & \multirow{5}{*}{.0001} \\
\hline & Skin scrapping & $10.20 \pm 0.81$ & \\
\hline & Nasal secretion & $10.14 \pm 0.71$ & \\
\hline & Rectal swab & $10.15 \pm 0.703$ & \\
\hline & Feaces & $10.36 \pm 0.47$ & \\
\hline
\end{tabular}

Table 5:- Breed wise distribution of TBC of zoonotic bacterial pathogen from pet dogs.

[Where, All values are converted into logarithms 10; All counts are expressed in colony forming units (CFU); 0.0001 means statistically highly significant.]

\begin{tabular}{|c|c|c|c|c|c|c|c|c|}
\hline \multicolumn{2}{|c|}{$\begin{array}{c}\text { Serial } \\
\text { Test } \\
\text { Parameters }\end{array}$} & 1 & 2 & 3 & 4 & 5 & 6 & 7 \\
\hline \multirow{3}{*}{ SF } & Lac & AG & AG & - & - & - & $\mathbf{A}$ & - \\
\hline & Dex & AG & AG & AG & AG & - & $\mathbf{A}$ & $\mathbf{A}$ \\
\hline & Suc & $\mathbf{A}$ & AG & $\mathbf{A}$ & - & - & $\mathbf{A}$ & $\mathbf{A}$ \\
\hline \multicolumn{2}{|c|}{ Oxidase } & - & - & - & - & + & - & - \\
\hline \multicolumn{2}{|c|}{ Catalase } & + & + & + & + & + & + & - \\
\hline \multicolumn{2}{|r|}{ Indole } & + & - & - & + & - & - & - \\
\hline \multirow{2}{*}{\multicolumn{2}{|c|}{ MR Reaction }} & + & - & + & + & - & + & - \\
\hline \multirow{2}{*}{\multicolumn{2}{|c|}{$\frac{\text { VP Reaction }}{\text { SC }}$}} & - & + & - & + & - & - & + \\
\hline & & - & + & + & + & + & - & - \\
\hline \multicolumn{2}{|c|}{ Ornithine } & - & + & + & + & + & - & - \\
\hline \multicolumn{2}{|r|}{ TSI } & YY & YY & YR & YR & $\mathbf{R R}$ & YR & YR \\
\hline \multirow{2}{*}{\multicolumn{2}{|c|}{$\begin{array}{l}\text { MIU } \\
\text { Selenite }\end{array}$}} & + & - & + & + & + & + & + \\
\hline & & + & + & + & + & + & - & - \\
\hline \multicolumn{2}{|r|}{ Results } & E.coli & $\begin{array}{l}\text { Klebsiella } \\
\text { spp }\end{array}$ & $\begin{array}{l}\text { Salmonella } \\
\text { Spp }\end{array}$ & $\begin{array}{l}\text { Proteus } \\
\text { spp }\end{array}$ & $\begin{array}{c}\text { Pseudomonas } \\
\text { spp }\end{array}$ & $\begin{array}{c}\text { Staphylococcus } \\
\text { spp }\end{array}$ & $\begin{array}{c}\text { Streptococcus } \\
\text { Spp }\end{array}$ \\
\hline
\end{tabular}

Table 6:- Results of Biochemical Tests of isolated bacteria

[Where; A= Acid, G= Gas, $+=$ positive, $-=$ negative, $\mathrm{YY}=$ Yellow-yellow; YR= Yellow-Red, $\mathrm{SF}=$ Sugar Fermentation, Lac $=$ Lactose, $\mathrm{Dex}=$ Dextrose, Suc $=$ Sucrose, MR= Methyl Red, VP= Voges-Proskaur, SC= Simmons Citrate, TSI $=$ Triple Sugar Iron, MIO= Motility Indole Urease]

\section{Antibiotic Sensitivity Test}

On antibiogram study 18 antibiotics were used for antimicrobial sensitivity test. Gram negative isolates were more sensitive to Chloramphenicol, Cephalexin, Gentamycin and Kanamycin. On the other hand gram positive isolates were more sensitive to Azithromycin, Colistin, Gentamycin and Levofloxacin. On the other hand Azithomycin; Chloramphenicol were intermediate for gram negative bacteria. The all isolates were highly resistant to Amoxycillin, Bacitracin, Penicillin and Vancomycin. Gram positive isolates were more sensitive to Azithromycin, Colistin, Gentamycin and Levofloxacin.

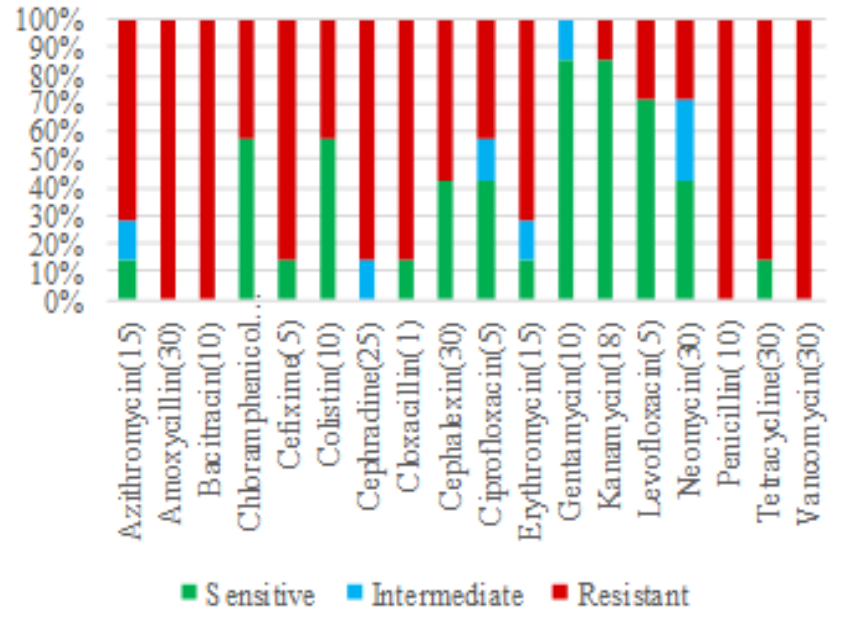

Fig 8:- Column diagram presenting antibiotic sensitivity test of isolated Bacteria 


\section{DISCUSSION}

The results of the current study revealed that the prevalence of different bacterial pathogen in relation to socio-demographic variable in case of housing system and hygienic condition were statistically significant $(\mathrm{P}<0.05)$ whereas in case of age, sex, body weight, diet and the educational quality of pet owners were not statistically significant $(\mathrm{P}>0.05)$.

The results of total bacterial count (TBC) are not statistically significant $(\mathrm{P}>0.05)$ among the different samples and pet animals showed in TABLE III. The TBC in oral swab, skin scraping, nasal swab, rectal swab and

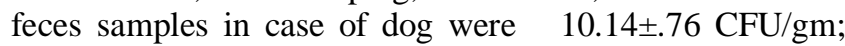
$10.16 \pm .78 \mathrm{CFU} / \mathrm{gm} ; \quad 10.14 \pm .75 \mathrm{CFU} / \mathrm{gm} ; 10.13 \pm .80$ $\mathrm{CFU} / \mathrm{gm} ; 10.35 \pm .54 \mathrm{CFU} / \mathrm{gm}$ respectively. In case of cat oral swab, skin scarping, nasal swab and rectal swab samples were $10.50 \pm .04 \mathrm{CFU} / \mathrm{gm} ; 10.57 \pm .057 \mathrm{CFU} / \mathrm{gm}$; $10.55 \pm .11 \mathrm{CFU} / \mathrm{gm}$ and $10.63 \pm .15 \mathrm{CFU} / \mathrm{gm}$ respectively. In case of rabbit oral swab, skin scarping, nasal swab, rectal

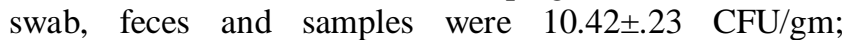
$10.41 \pm .24 \mathrm{CFU} / \mathrm{gm} ; 10.45 \quad \pm .28 \mathrm{CFU} / \mathrm{gm} ; 10.47 \pm .26$

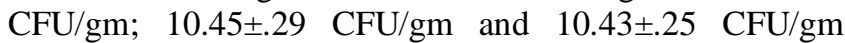
respectively. Species, sex and age wise distribution of total viable count of zoonotic bacterial pathogen from pet animals showed in TABLE IV. This value analyzed by one way ANOVA test and these values are not statistically significant $(\mathrm{P}>0.05)$. Species and breed wise distribution of total viable count of zoonotic bacterial pathogen from household pet dogs showed in TABLE $\mathrm{V}$ and all the values are statistically significant $(\mathrm{P}<0.01)$. The overall prevalence of isolated bacteria in case of dog out of 250 samples were $15.2 \%$ Escherichia coli; $10.8 \%$ Klebsiella spp; $12 \%$ Salmonella spp; $8 \%$ Proteus spp; $8.8 \%$ Pseudomonas spp; $12.8 \%$ Staphylococcus spp and $10.4 \%$ Streptococcus spp respectively. The overall prevalence of isolated bacteria in case of cat out of 40 samples were $17.5 \%$ Escherichia coli; 10\% Klebsiella spp; $12.5 \%$ Salmonella spp; $10 \%$ Proteus spp; 10\% Pseudomonas spp; 15\% Staphylococcus spp and $15 \%$ Streptococcus spp respectively. The overall prevalence of isolated bacteria in case of rabbit out of 114 samples were $12.28 \%$ Escherichia coli; $7.89 \%$ Klebsiella spp; $11 \%$ Salmonella spp; 5\% Proteus spp; $2 \%$ Pseudomonas spp; 6.14\% Staphylococcus spp and 6.14\% Streptococcus spp respectively. Isolated E. coli, Pseudomonas spp, Proteus spp and Staphylococcus spp from dog and cat which commonly causes infectious diseases and transmitted from animal to human by direct contact [16]. Prevalence of Salmonella ranging from 0-9\% and $0-4 \%$ in dogs and cats respectively and higher prevalence may be found in stray or shelter dogs/cats [17]. This organism can be transmitted directly or indirectly by the fecal-oral rout and develops the symptoms of gastroenteritis in human [18]. In a study conducted by Hashemi, reported that the prevalence rate of Salmonella were 18 and $22 \%$ in cat and respectively which agree with the current study [19]. Another one reported that the prevalence of Salmonellosis in dog and cat were $23.15 \%$ and $13.05 \%$ respectively in Dhaka, Bangladesh [20]. E.coli are the part of the normal intestinal micro-flora, but they can cause gastroenteritis when local or systemic immunity does not work properly. Enteropathogenic strains of $E$. coli (EPEC) have been found in human patients and dogs that live in the same household [21]. Recent findings indicate that the transmission of diarrheagenic E. coli strains occurs between dogs and human. Asymptomatic dogs were identified as carrier of human pathogenic Shiga-toxinproducing (STEC) E. coli play an important role in outbreaks of STEC infections in human [22] [23]. Diarrheic dogs were identified as an important source of bacterial contamination of the environment in apartments of dog holders that might contribute to the spread and transmission of pathogenic E. coli strains [24]. Staphylococcus spp in a human source bacteria primarily in our anterior nares and on hands, cats and less commonly dogs may carry $S$. aures in their normal skin and mucosal bacterial flora [25]. But S. pseudintermediusis a dog or cat source bacteria found in cases of pyoderma, otitis and wound infections. This species is the most common cause of Staphylococcus infections in pets and also a major cause of dog bite infection in people. Belli reported that the prevalence rate of diluent bacterial isolates in rabbit were Streptococcus spp (22.8\%) Staphylococcus spp (17.8\%), E.coli (12.5\%), proteus spp $(5.7 \%)$ which supports the findings of the current study [26]. On the other hand, Martino and Luzi found that the prevalence of Klebsiella spp; Pseudomonas spp; Staphylococcus spp; Streptococcus spp and Escherichia coli were 9.4\%, 15.6\%, 6.2\%, 3.1\% and $3.1 \%$ respectively in rabbit. These findings are as agreed with the results of the current study [7]. Okumu isolated that Escherichia coli and Staphylococcus aureus were frequently isolated from conjuctival and nasopharyngeal swabs were $4.17 \%$ and $8.33 \%$ respectively which findings are also agreement with the results of the current study [27].

Martino described that samples came mainly from nose (swabs) with a prevalence of $37.5 \%$, then from abscesses or abdominal cavity $(28.1 \%)$ and from eyes (15.6\%). Pasteurella multocida was the most isolated bacterium (21.9\%) followed by Pseudomonas aeruginosa $(15.6 \%)$ and Klebsiella pneumonia (9.4\%). The other microorganisms were isolated in a low percentage. Many samples $(28 \%)$ were negative probably due to sampling mistakes or to the poor significance of the collected sample (e.g, insufficient drawn material or sampling not in the correct site) or to antibiotic treatment of animals just before sampling. These data are in agreement with the high spreading of these types of pathologies in pet rabbits as reported by current study [7]. Dogs have been reported to be the carrier of Salmonella spp worldwide which have the potential to serve as sources of exposure or infection for humans [28]. It was reported that the intestinal carriage of salmonellae by dogs is more common than the prevalence of clinical disease. The frequency of faecal isolation of Salmonella spp. from clinically healthy dogs was reported to be between $0.0 \%$ and $43.0 \%$ [28] [29]. The current study found $12 \%$ positive result for Salmonella spp from dog. 
On antibiogram study 18 antibiotics were used against 7 isolated bacteria for antimicrobial sensitivity test. Cephalexin and kanamycin were $100 \%$ sensitive for all gram negative isolates. Whereas Chloramphenicol was 100\% sensitive to E.coli; Klebsiella spp; Proteus spp and Pseudomonas spp but $66.7 \%$ resistant to Salmonella spp. On the other hand ciprofloxacin was $100 \%$ sensitive for Klebsiella spp and Proteus spp but $100 \%$ resistant to E. coli; Salmonella spp and Pseudomonas spp. Again erythromycin was $100 \%$ sensitive to Pseudomonas spp but $66.7 \%$ resistant and $33.3 \%$ intermediate to E.coli; Klebsiella spp; Proteus spp and Salmonella spp. Gentamycin was $66.7 \%$ intermediate to Salmonella spp but $100 \%$ sensitive to E.coli; Klebsiella spp; Proteus spp and Pseudomonas spp. Neomycin was $100 \%$ sensitive to E.coli and Proteus spp. Azithromycin, colistin, gentamycin and levofloxacin were $100 \%$ sensitive to Staphylococcus spp. Chloramphenicol, cephradine, ciprofloxacin, erythromycin, neomycin, kanamycin and cephalexin were $66.7-100 \%$ resistant to Staphylococcus spp. Amoxycillin, bacitracin, cefixime, cloxacillin, penicillin, tetracycline and vancomycin were $100 \%$ resistant to all isolates. According to Gerding, the most effective antibiotics for Staphylococcus spp. were bacitracin, gentamicin and tobramycin; while chloramphenicol and erythromycin for Streptococcus spp. Prado, showed that $80.7 \%$ of the isolates were gram positive cocci and gram positive bacilli, and those species were sensitive to gentamicin, ciprofloxacin, chloramphenicol and tobramycin. This result is agreed with the findings of the current study [30] [31]. Staphylococcus species isolated from dogs with pyoderma were found to be resistant to streptomycin, kanamycin, neomycin and erythromycin (28\%), to clindamycin (22\%) and to gentamicin and enrofloxacin [32]. Keskin reported that $82.5 \%$ of the bacteria isolated from the dogs with otitis externa were resistant to enrofloxacin, $65.5 \%$ to cephalosporins, $44.4 \%$ to gentamicin and tetracycline, $34.9 \%$ to spiramycin, 26.9 to ampicillin, while $20.6 \%$ were resistant to lincomycin [33]. Sarierler reported that bacteria isolated from dogs with otitis externa were resistant to oxytetracycline $(100 \%)$, ciprofloxacin $(100 \%)$, kanamycin $(87.5 \%)$, penicillin $\mathrm{G}(72.5 \%)$, erythromycin $(57.5 \%)$, gentamicin (55\%), ampicillin (50\%) and cefoperazone (50.0\%). Keskin was reported that Staphylococcus species were highly resistant $(63.1 \%)$ to ampicillin. Hariharan reported that most isolates were susceptible to gentamycin but resistant to ampicillin, penicillin. Schick reported that most isolates were susceptible to gentamycin (81\%) and enrofloxacin $(56 \%)$. Keskin reported that most bacterial strains from dogs were sensitive to enrofloxacin, gentamycin, and lincomycin, but resistant to tetracycline and ampicillin. Martin reported that most isolates were susceptible to tobramycin (100\%), marbofloxacin (90\%), ceftazidime $(90 \%)$, gentamycin $(68 \%)$, and enrofloxacin $(42 \%)$. All finding are nearly agreement with the results of the current study [33] [34] [35] [36] [37].

\section{CONCLUSIONS}

Since the isolation bacteria can be potentially pathogenic to people, this study comes to demonstrate that there is on the contact of human to household pets, despite the absence of the clinical signs and symptoms from the zoonotic bacterial infection. Also, children may snuggle with pets can increase the risk zoonotic disease transmission. Hence the owner of the pets should have correct knowledge about zoonoses for their prevention and should take precautionary measures, improve personal hygienic to reduce the risk contact with pathogenic bacteria.

\section{ACKNOWLEDGMENT}

Authors are very thankful to all staff of Department of Microbiology, Faculty of Veterinary and Animal Science (FVAS), Hajee Mohammad Danesh Science and Technology University (HSTU) for their cooperation and supporting laboratory works.

\section{REFERENCES}

[1]. B. Ryoko, T. Maeda, M. Kamei \& Kourai "Pathogenic bacteria carried by companion animals and their susceptibility to antibacterial agents" Biocontrol Science, 11(1), 1-9, 2006.

[2]. D.F. Morey "The early evolution of the domestic dog" Sci. Am. 82: 336-347, 1994.

[3]. M.A. Parvez, M.A.M Prodhan, B.C. Das \& R. Khater "Prevalence of clinical conditions in dogs and cats at teaching veterinary hospital (TVH) in Chittagong Veterinary and Animal Science University, Bangladesh" Res J Vet Prac, 2(6), 99-104, 2014.

[4]. I.R. Dohoo, W.N. McDonell, C.S. Rhodes \& Y.L. Elazhary, "Veterinary research and human health" Can. Vet. J., 39, 549-556, 1998.

[5]. I.D. Robertson, P.J. Irwin, A.J. Lymbery \& R.C.A Thompson "The role of companion animals in the emergence of parasitic disease" Intern. J. Parasitol., 30, 1369-1377, 2000.

[6]. PDSA "PDSA Animal wellbeing Report. Available from: https://www.pdsa.org.uk/get-involved/ourcurrent-campaigns/pdsa-animal-wellbeing-report" 2015.

[7]. P.A. Martino \& F. Luzi "Bacterial infections in rabbit as companion animal: A survey of diagnostic samples in Italy" Pathol. Hyg. 1013-1018, 2008.

[8]. J.C. Dey, M.K. Rahman, M.A. Rumi, A. Dutta, M.A. Sayeed, B.C. Halder, A. Mannan \& M.S. Hossain "Prevalence of dermatophytosis in rabbits at SAQTUH, Chittagong, Bangladesh" J Dairy Vet Anim Res, 3(6), 1-5, 2016.

[9]. B. C. Bruno "Emerging and Re-Emerging Zoonoses of Dogs and Cats". Ani. J., 4, 434-445, 2014.

[10]. WHO/FAO expert committee on zoonoses "World Health Organization Technical Report Series", 58, 184, 1959. 
[11]. Anon "Zoonoses http://en.wilipedia.org/wiki/zoonosis" 2011.

[12]. S.J. Song, C. Lauber, E.K. Costello, C.A. Lozupone \& Humphrey G. "Cohabiting family members share microbiota with one another and with their dogs" eLife, 2, e00458, 2013.

[13]. M. Varga "Questions around Encephalitozoon cunuculi in rabbits" Veterinary Record 174: 347-348, 2014.

[14]. M. Kantere, L.V. Athanasiou, D.C. Chatzopoulos, V. Spyrou, G. Valiakos, V. Kontos \& C. Billinis "Enteric pathogens of dogs and cats with public health implications” Am. J. Ani. Vet. Sci.,9(2), 84-94, 2014.

[15]. CLSI - Clinical and Laboratory Standards Institute (2011): Performance standards for antimicrobial susceptibility testing: Twentieth informational supplement M100-S20. CLSI, Wayne, PA, USA.

[16]. R. Buma, T. Maeda, M. Kamei \& H. Kourai "Pathogenic bacteria carried by companion animals and their susceptibility to antibacterial agents" Biocon. Sci., 11(1), 1-9, 2006.

[17]. S.L. Marks, S.C. Rankin, B.A. Byrne \& J.S. Weese "Enteropathogenic Bacteria in Dogs and Cats: Diagnosis, Epidemiology, Treatment and Control" JVIM, 25 (6), 1195-1208, 2011.

[18]. P. Damborg, E.M. Broens, B.B. Chomel, S. Guenther, F. Pasmans, J. A. Wagenaar, J.S. Weese, L. H. Wieler, U. Windahl, D. Vanrompay \& L. Guardabassi "Bacterial zoonoses transmitted by household". Tar. Res.Poli. Acti., 155, 27 - 40, 2016.

[19]. S. Hashemi, M. Mahzounieh \& M. Ghorbari "Detection of Yersinia spp and Salmonella spp in apparently healthy cats and dogs in Tehran, Iran" Biol. J. Microor., 4(16), 49-54, 2016.

[20]. S.S.M.R. Hossain \& M.E.H. Kayesh "Common disease of pet animals in Dhaka city and their zoonotic importance" Intern. J. Nat. Soci. Sci., 1, 81-84, 2014.

[21]. J. Rodrigues, C.M. Thomazini, C.A. Lopes \& L.O. Dantas "Concurrent infection in a dog and colonization in a child with a human enteropathogenic Escherichia coli clone" J. Clin. Microbiol, 42, 13881389, 2004.

[22]. R. Khakhria, D. Duck \& H. Lior "Extended phagetyping scheme for Escherichio cnli 0157:H7" Epidemiol. Infect. 105, 511-520, 1990.

[23]. W.B. Trevena, R.S. Hooper, C. Wray, G.A. Willshaw, T. Cheasty \& G. Domingue "Vero cytotoxin producing Escherichia coli O 157 associated with companion animals" Vet. Rec., 138, 400, 1996.

[24]. A. Mayr, S. Goetz \& H. Schels "Untersuchungen ueber die Hygiene bei der Haltung von Hunden in staedtischen Wohnungen (The hygiene of keepingdogs in city apartments), Zentralbl. Baktcriol" Mikrobiol. Hyg. Ser. B, Umwelthyg. Krankenhaushyg. Arbeitshyg. Praev. Med., 183, 1986.

[25]. G.C. Griffeth "Screening for skin carriage of methicillin-ressitant coagulase-positive Staphylococci and Staphylococcus schleiferi in dogs with healthy and inflamed skin" Vet Derm., 19; 142-149, 2008.
[26]. P. Belli, E. Fontana, M. Sommariva, L. Scarpelli, C. Ricci, F. Luzi \& B. Haddad "The tunisian traditional rabbit breeding system versus the commercial system: an epidemiological perspective" World Rabbit Sci., 16, 221- 228, 2008.

[27]. P.O. Okumu, P.K. Gathumbi, D.N. Karanja, L.C. Bebora, J.D. Mande, J.K. Serem, M.M. Wanyoike, C. Gachuiri, R.N. Mwanza \& S.K. Mailu "Survey of Health Status of Domestic Rabbits in Selected Organized Farms in Kenya" Intern. J. Vet. Sci., 4(1), 15-21, 2014.

[28]. M.E. Carter \& J.P. Quinn "Salmonella infections in dogs and cats" In: Wray, C., and A. Wray (eds) Salmonella in Domestic Animals, CAB International, Wallingford, UK, 231-244, 2000.

[29]. S. Sanchez, C.L. Hofacre, M.D. Lee, J.J. Maurer, M.P. Doyle (2002). Animal sources of salmonellosis in humans. javma 221(4), 492-497.

[30]. P.A. Gerding, S.A. Mclaughlin \& M.W. Troop "Pathogenic bacteria and fungi associated with external diseases in dogs: 131 cases (1981-1986)" J. Am. Vet. Med. Assoc., 193, 242-244, 1988.

[31]. M.R. Prado, E.H.S. Brito, M.D. Girao, J.J.C. Sidrim \& M.F.G. Rocha "Identification and antimicrobial susceptibility of bacteria isolated from corneal ulcers of dogs" Arq. Bras. Med. Vet. Zootec, 58(6), 1024 1029, 2006.

[32]. M. Boost, M. O’Donoghue \& A. James “Investigation of the role of $\operatorname{dog}$ s as reservoirs of Staphylococcus aureus and the transmission of strains between pet owners and their dogs" Hong Kong Med. J., 14, 15$18,2008$.

[33]. O. Keskin, L. Kokcu \& M. Akan "Identification and antimicrobial sensitivity of microorganisms isolated from otitic dogs" Vet. J. Ankara Univ. 46, 163-168, 1999.

[34]. M. Sarierler \& S. Kirkan "Microbiological diagnosis and therapy of canine otitis externa" Vet. Cerrahi Dergisi 10, 11-15, 2004.

[35]. H. Hariharan, M. Coles, D. Poole, L. Lund \& R. Page "Update on antimicrobial susceptibilities of bacterial isolates from canine and feline otitis externa" Can. Vet. J., 47, 253-255, 2006.

[36]. A.K. Schick, J.C. Angus \& K.S. Coyner "Variability of laboratory identification and antibiotic susceptibility reporting of Pseudomonas spp. isolates from dogs with chronic otitis externa" Vet. Derm, 18, 120-126, 2007.

[37]. B.J.L. Martin, G.P. Lupiola, L.Z. Gonzalez \& J.M.T. Tejedor "Antimicrobial susceptibility patterns of Pseudomonas strains isolated from chronic canine otitis externa" J. Vet. Med. B Infect. Vet. Pub H., 47, 191-196, 2000. 